\title{
A dual-mixed finite element method for quasi-Newtonian flows whose viscosity obeys a power law or the Carreau law
}

\author{
Mohamed Farhloul $^{\mathrm{a}}$, Abdelmalek Zine ${ }^{\mathrm{b}}$ \\ ${ }^{a}$ Département de Mathématiques et de Statistique, Université de Moncton, Moncton, N.B., E1A 3E9, Canada \\ ${ }^{b}$ Université de Lyon, Institut Camille Jordan, CNRS-UMR5208, Département de Mathématiques et Informatique, \\ Ecole Centrale de Lyon, 36 av. Guy de Collongue, 69134 Ecully Cedex, France
}

\begin{abstract}
The aim of this work is a construction of a dual mixed finite element method for a quasi-Newtonian flow obeying the Carreau or power law. This method is based on the introduction of the stress tensor as a new variable and the reformulation of the governing equations as a twofold saddle point problem. The derived formulation possesses local (i.e. at element level) conservation properties (conservation of the momentum and the mass) as for finite volume methods. Based on such a formulation, a mixed finite element is constructed and analyzed. We prove that the continuous problem and its approximation are well posed, and derive error estimates.
\end{abstract}

AMS (MOS) subject classification 65N30; 65N15;

Keywords: Mixed Finite Element, Quasi-Newtonian, Power law, Carreau law

\section{Introduction}

We propose a dual-mixed formulation for non-Newtonian fluid flow where the fluid viscosity is assumed to be nonlinear function of the rate of strain tensor. The governing equations arise in modeling flows of, for example, biological fluids, lubricants, paints and polymeric fluids. In $[9,10]$ we have introduced and analyzed a dual-mixed finite element method for quasi-Newtonian fluid flow obeying to the power law. A priori error estimates for the finite element approximation were proved in [9], while a posteriori error estimation was provided in [10]. However, in both [9, 10] the analysis used the assumption that the equation describing the stress tensor in terms of the rate of strain tensor was invertible to give the rate of strain tensor as function of the stress tensor. The mixed finite element method developed in [9] possesses local (i.e., at element level) conservation properties (conservation of the momentum and the mass) as in the finite volume methods. Furthermore, it allows the approximations of all the physical variables (stress, velocity and pressure).

The aim of this work is to extend our investigations by avoiding the assumption of expressing the rate of strain tensor as function of the stress tensor. As example of such a situation is a nonNewtonian fluid flow obeying the Carreau law. For this purpose, we introduce an additional variable for the rate of strain tensor and reformulate the governing equations as a twofold saddle point problem. It must be noted that this kind of approach has been introduced and analyzed in $[12,7]$ for a class of quasi-Newtonian Stokes flows. However, in both $[12,7]$ the tensor gradient of the velocity was used instead of the rate of strain tensor. The fact to use the rate of strain tensor introduces a major difficulty in the construction of mixed finite element methods (for more details, see [4]). The difficulty lies essentially in the symmetry of this tensor. One way to overcome this difficulty is to relax the symmetry of this tensor by a Lagrange multiplier. We will present our dual-mixed formulation and establish well posedness. The mixed finite element for this formulation will be provided

Email address: Mohamed.Farhloul@UMoncton.ca, Abdel-Malek.Zine@ec-lyon.fr (Abdelmalek Zine) 
and the associated a priori error estimates are then derived. The error estimates are optimal and are the same as the ones obtained in the particular case of Power law ([9]).

The outline of the paper is as follows: In Section 2 the governing equations and the mixed formulation of non Newtonian flows whose viscosity obeys a power law or the Carreau law are presented. Existence and uniqueness results are given in Section 3. In Section 4 we introduce our finite element approximation and establish well posedness. The a priori error estimates are then derived and last section is devoted to the conclusion.

\section{Governing equations and mixed formulation}

Governed by the classical Stokes problem, the Newtonian fluid flows are a reasonable approximation of the more realistic non-Newtonian fluids (quasi-Newtonian or Viscoelastic). In the case of quasi-Newtonian fluids, the viscosity is a function of gradient tensor, temperature, time, etc. For a steady and creeping flow of an incompressible quasi-Newtonian fluid, the most used formulation is based on the strain rate tensor.

In that case, for $\Omega$ a bounded domain of $\mathbb{R}^{2}$ with a Lipschitz boundary $\Gamma$ and a given mass forces $f$ defined in $\Omega$, the combination of the conservation equations leads to the following Nonlinear Stokes problem:

$$
\begin{cases}-\boldsymbol{d i v}(2 \nu(|\boldsymbol{d}(\boldsymbol{u})|) \boldsymbol{d}(\boldsymbol{u}))+\nabla p=\boldsymbol{f} & \text { in } \Omega \\ \operatorname{div} \boldsymbol{u}=0 & \text { in } \Omega\end{cases}
$$

where $\boldsymbol{u}$ and $p$, the unknowns of the problem, are the velocity and pressure, respectively. $\boldsymbol{d}(\boldsymbol{u})=$ $\frac{1}{2}\left(\nabla \boldsymbol{u}+(\nabla \boldsymbol{u})^{t}\right)$ is the strain rate tensor, and $|\boldsymbol{d}(\boldsymbol{u})|^{2}=\sum_{i, j=1}^{2} \boldsymbol{d}(\boldsymbol{u})_{i j}^{2}$.

For $\nu_{0}>0$ a reference viscosity and $r$ a fluid characteristic real parameter verifying $1<r<\infty$, the viscosity function $\nu(\cdot)$, depending on $|\boldsymbol{d}(\boldsymbol{u})|$, is usually given by one of the two following famous models:

$$
\begin{aligned}
& \nu(x)=\nu_{0} x^{r-2}, \forall x \in \mathbb{R}_{+}, \text {for the Power law model, and } \\
& \nu(x)=\nu_{0}\left(1+x^{2}\right)^{(r-2) / 2}, \forall x \in \mathbb{R}_{+}, \text {for the Carreau model. }
\end{aligned}
$$

Finally, system (1) is supplemented by a set of boundary conditions.

Remark 2.1. For $r=2$, both models provide the well known classical Stokes problem:

$$
\left\{\begin{array}{rlll}
-2 \nu_{0} \boldsymbol{d i v}(\boldsymbol{d}(\boldsymbol{u}))+\nabla p & = & \boldsymbol{f} & \text { in } \Omega \\
\operatorname{div} \boldsymbol{u} & = & 0 & \text { in } \Omega,
\end{array}\right.
$$

corresponding to a Newtonian fluid flow.

The generalized Stokes problem (1) and its approximation by standard finite elements was first studied in Baranger and Najib [1]. Extensions and improvements of the error bounds have been obtained in Sandri [17] and Barrett and Liu [2,3].

In these works, only the primal variables velocity and pressure are taken into account. But, for various reasons, we need information on other variables as velocity gradients $\nabla \boldsymbol{u}$, strain rate tensor $\boldsymbol{d}(\boldsymbol{u})$, extra-stress tensor $\boldsymbol{\sigma}=2 \nu(|\boldsymbol{d}(\boldsymbol{u})|) \boldsymbol{d}(\boldsymbol{u})$ etc. In that case, it is necessary to build appropriate mixed formulations.

On the other hand, in connection with the use of the gradient tensor $\nabla \boldsymbol{u}$ which corresponds to the Ladyzhenskaya model [14]:

$$
\nu(|\nabla \boldsymbol{u}|)=\left(\nu_{0}+\nu_{1}|\nabla \boldsymbol{u}|\right)^{r-2}, \nu_{0} \geq 0, \nu_{1}>0, r>1,
$$


a large amount of work is available in the literature. Among these works, there may be mentioned Manouzi and Farhloul [15], Farhloul and Zine [8], Gatica et al. [11, 12] and Ervin et al. [7].

The major drawback of formulations using the gradient lies in the fact that we can not deal with natural boundary conditions. To overcome this drawback related to the boundary conditions, we have introduced and analyzed a dual-mixed finite element method for quasi-Newtonian fluid flow obeying to the Power law, in Farhloul and Zine $[9,10]$. A priori error estimates for the finite element approximation were proved in the first paper, while a posteriori error estimation was provided in the second work. In both, the analysis is based on the fact that the equation describing the extra-stress tensor in terms of the rate of strain tensor:

$$
\boldsymbol{\sigma}=|\boldsymbol{d}(\boldsymbol{u})|^{r-2} \boldsymbol{d}(\boldsymbol{u}) \quad\left(\nu_{0}=1 / 2\right),
$$

is invertible and give the rate of strain tensor as a function of the stress tensor,

$$
\boldsymbol{d}(\boldsymbol{u})=|\boldsymbol{\sigma}|^{r^{\prime}-2} \boldsymbol{\sigma}
$$

$r^{\prime}$ being the conjugate of $r$.

The aim of this work is to extend our investigations by avoiding the assumption of expressing the rate of strain tensor as function of the stress tensor. We may be then able to deal with both problems associated with Power law and Carreau Model.

In order to obtain a dual-mixed formulation of (1), we first formulate problem (1) as:

$$
\begin{cases}-\boldsymbol{d i v}(\boldsymbol{\sigma}-p \boldsymbol{I})=\boldsymbol{f} & \text { in } \Omega, \\ \operatorname{div} \boldsymbol{u}=0 & \text { in } \Omega, \\ \boldsymbol{u}=0 & \text { on } \Gamma,\end{cases}
$$

and then, we introduce two new variables

$$
\begin{aligned}
\boldsymbol{t} & =\boldsymbol{d}(\boldsymbol{u}), \text { the strain rate tensor, } \\
\mathcal{A}(\boldsymbol{t}) & =2 \nu(|\boldsymbol{t}|) \boldsymbol{t}=\boldsymbol{\sigma}, \text { the extra stress tensor. }
\end{aligned}
$$

Suppose $\boldsymbol{f} \in\left[L^{r}(\Omega)\right]^{2}, 1<r<\infty$. Let $r^{\prime}$ being the conjugate number of $r$, i.e. $\frac{1}{r}+\frac{1}{r^{\prime}}=1$. Let $\boldsymbol{\omega}=\boldsymbol{\omega}(\boldsymbol{u})=\frac{1}{2}\left(\nabla \boldsymbol{u}-(\nabla \boldsymbol{u})^{t}\right)$ be the vorticity tensor. Then, for all $(\boldsymbol{\tau}, q) \in\left[L^{r^{\prime}}(\Omega)\right]^{2 \times 2} \times L_{0}^{r^{\prime}}(\Omega)$, such that $\operatorname{div}(\boldsymbol{\tau}-q \boldsymbol{I}) \in\left[L^{r^{\prime}}(\Omega)\right]^{2}$, and for all $\boldsymbol{u} \in W^{1, r}(\Omega)$ such that $\operatorname{div} \boldsymbol{u}=0$, it is easy to see that:

$$
(\boldsymbol{t}, \boldsymbol{\tau})=(\boldsymbol{d}(\boldsymbol{u}), \boldsymbol{\tau}),=-(\operatorname{div}(\boldsymbol{\tau}-q \boldsymbol{I}), \boldsymbol{u})-(\boldsymbol{\omega}, \boldsymbol{\tau}),
$$

where, from now on, $(\cdot, \cdot)$ denotes the duality pairing between $L^{r^{\prime}}(\Omega)$ and $L^{r}(\Omega)$. The space $L_{0}^{r^{\prime}}(\Omega)$ is defined by

$$
L_{0}^{r^{\prime}}(\Omega)=\left\{q \in L^{r^{\prime}}(\Omega) ; \int_{\Omega} q d x=0\right\} .
$$

In order to derive the mixed formulation of (2), we define the following spaces:

$$
\begin{aligned}
\boldsymbol{T} & =\left[L^{r}(\Omega)\right]^{2 \times 2}, \\
\boldsymbol{\Sigma} & =\left\{(\boldsymbol{\tau}, q) \in\left[L^{r^{\prime}}(\Omega)\right]^{2 \times 2} \times L_{0}^{r^{\prime}}(\Omega) ; \boldsymbol{d i v}(\boldsymbol{\tau}-q \boldsymbol{I}) \in\left[L^{r^{\prime}}(\Omega)\right]^{2}\right\}, \\
\boldsymbol{M} & =\left\{(\boldsymbol{v}, \boldsymbol{\eta}) \in\left[L^{r}(\Omega)\right]^{2} \times\left[L^{r}(\Omega)\right]^{2 \times 2} ; \boldsymbol{\eta}+\boldsymbol{\eta}^{t}=0\right\},
\end{aligned}
$$

equipped with the following norms:

$$
\begin{aligned}
\|\boldsymbol{s}\|_{T} & =\|\boldsymbol{s}\|_{0, r, \Omega}=\left(\int_{\Omega}|\boldsymbol{s}|^{r}\right)^{\frac{1}{r}} \\
\|(\boldsymbol{\tau}, q)\|_{\Sigma} & =\left(\|\boldsymbol{\tau}\|_{0, r^{\prime}, \Omega}^{r^{\prime}}+\|q\|_{0, r^{\prime}, \Omega}^{r^{\prime}}+\|\boldsymbol{d i v}(\boldsymbol{\tau}-q \boldsymbol{I})\|_{0, r^{\prime}, \Omega}^{r^{\prime}}\right)^{\frac{1}{r^{\prime}}} \\
\|(\boldsymbol{v}, \boldsymbol{\eta})\|_{M} & =\left(\|\boldsymbol{v}\|_{0, r, \Omega}^{r}+\|\boldsymbol{\eta}\|_{0, r, \Omega}^{r}\right)^{\frac{1}{r}}
\end{aligned}
$$


The dual-mixed formulation of problem (2) reads as follows: Find $\boldsymbol{t} \in \boldsymbol{T},(\boldsymbol{\sigma}, p) \in \boldsymbol{\Sigma}$ and $(\boldsymbol{u}, \boldsymbol{\omega}) \in \boldsymbol{M}$ such that

$$
\begin{cases}(\mathcal{A}(\boldsymbol{t}), \boldsymbol{s})-(\boldsymbol{\sigma}, \boldsymbol{s})=0 & \forall \boldsymbol{s} \in \boldsymbol{T}, \\ (\boldsymbol{t}, \boldsymbol{\tau})+(\boldsymbol{d i v}(\boldsymbol{\tau}-q \boldsymbol{I}), \boldsymbol{u})+(\boldsymbol{\tau}, \boldsymbol{\omega})=0 & \forall \underset{\sim}{\boldsymbol{\tau}}=(\boldsymbol{\tau}, q) \in \boldsymbol{\Sigma}, \\ (\boldsymbol{d i v}(\boldsymbol{\sigma}-p \boldsymbol{I}), \boldsymbol{v})+(\boldsymbol{\sigma}, \boldsymbol{\eta})+(\boldsymbol{f}, \boldsymbol{v})=0 & \forall \underset{\sim}{\boldsymbol{v}}=(\boldsymbol{v}, \boldsymbol{\eta}) \in \boldsymbol{M} .\end{cases}
$$

Remark 2.2. From the last equation of (5),

$$
(\boldsymbol{d i v}(\boldsymbol{\sigma}-p \boldsymbol{I}), \boldsymbol{v})+(\boldsymbol{\sigma}, \boldsymbol{\eta})+(\boldsymbol{f}, \boldsymbol{v})=0, \forall(\boldsymbol{v}, \boldsymbol{\eta}) \in \boldsymbol{M},
$$

one gets

$$
(\boldsymbol{\sigma}, \boldsymbol{\eta})=0, \forall \boldsymbol{\eta} \in\left[L^{r}(\Omega)\right]^{2 \times 2} \text { such that } \boldsymbol{\eta}+\boldsymbol{\eta}^{t}=0 .
$$

This corresponds to the symmetry relaxation of the extra-stress tensor $\boldsymbol{\sigma}$ by a Lagrange multiplier.

Remark 2.3. As stated above, the use of the rate of strain tensor enable to handle different type of boundary conditions, such as mixed boundary conditions. More precisely, assuming that we consider the following boundary conditions:

$$
\boldsymbol{u}=0 \text { on } \Gamma_{D} \text { and }(2 \nu(|\boldsymbol{d}(\boldsymbol{u})|) \boldsymbol{d}(\boldsymbol{u})-p \boldsymbol{I}) \boldsymbol{n}=0 \text { on } \Gamma_{N},
$$

where $\Gamma=\Gamma_{D} \cup \Gamma_{N}, \Gamma_{D} \neq \emptyset$ and $\boldsymbol{n}$ is the unit outward normal vector field along the boundary of $\Omega$. Then, the only change to be made is to replace the space $\boldsymbol{\Sigma}$ by the following one:

$$
\boldsymbol{\Sigma}=\left\{(\boldsymbol{\tau}, q) \in\left[L^{r^{\prime}}(\Omega)\right]^{2 \times 2} \times L_{0}^{r^{\prime}}(\Omega) ; \boldsymbol{d i v}(\boldsymbol{\tau}-q \boldsymbol{I}) \in\left[L^{r^{\prime}}(\Omega)\right]^{2} ;(\boldsymbol{\tau}-q \boldsymbol{I}) \boldsymbol{n}=0 \text { on } \Gamma_{N}\right\} .
$$

However, we choose to make the analysis in the case of Dirichlet boundary conditions only for the sake of clearness.

To formally rewrite (5) as a twofold saddle-point problem, see Gatica et al. [11, 12], we define the following operators:

$$
\mathcal{A}: \boldsymbol{T} \longrightarrow \boldsymbol{T}^{\prime}, \mathcal{B}: \boldsymbol{T} \longrightarrow \boldsymbol{\Sigma}^{\prime} \text { and } \mathcal{C}: \boldsymbol{\Sigma} \longrightarrow \boldsymbol{M}^{\prime}
$$

where for a Banach space $\boldsymbol{X}, \boldsymbol{X}^{\prime}$ denotes the dual space with associated norm $\|\cdot\|_{X^{\prime}}$.

$$
\begin{aligned}
{[\mathcal{A}(\boldsymbol{t}), \boldsymbol{s}] } & =(\mathcal{A}(\boldsymbol{t}), \boldsymbol{s}), \forall \boldsymbol{s}, \boldsymbol{t} \in \boldsymbol{T} \\
{[\mathcal{B}(\boldsymbol{s}), \underset{\sim}{\boldsymbol{\tau}}] } & =-(\boldsymbol{s}, \boldsymbol{\tau}), \forall \boldsymbol{s} \in \boldsymbol{T}, \forall \underset{\sim}{\boldsymbol{\tau}}=(\boldsymbol{\tau}, q) \in \boldsymbol{\Sigma}, \\
{[\mathcal{C}(\underset{\sim}{\boldsymbol{\tau}}), \underset{\sim}{\boldsymbol{v}}] } & =-(\boldsymbol{d i v}(\boldsymbol{\tau}-q \boldsymbol{I}), \boldsymbol{v})-(\boldsymbol{\tau}, \boldsymbol{\eta}), \forall \underset{\sim}{\boldsymbol{\tau}} \in \boldsymbol{\Sigma}, \underset{\sim}{\boldsymbol{v}} \in \boldsymbol{M} .
\end{aligned}
$$

Remark 2.4. Recall that the operator $\mathcal{A}$ is defined by $\forall \boldsymbol{t} \in \boldsymbol{T}, \mathcal{A}(\boldsymbol{t})=2 \nu(|\boldsymbol{t}|) \boldsymbol{t}, \nu$ being given by either Power or Carreau law.

The problem (5) can be then written in the following twofold saddle-point form: Find $\boldsymbol{t} \in \boldsymbol{T}, \underset{\sim}{\boldsymbol{\sigma}} \in \boldsymbol{\Sigma}$ and $\underset{\sim}{\boldsymbol{u}} \in \boldsymbol{M}$ such that

$$
\begin{cases}{[\mathcal{A}(\boldsymbol{t}), \boldsymbol{s}]+\left[\boldsymbol{s}, \mathcal{B}^{\prime}(\underset{\sim}{\boldsymbol{\sigma}})\right]=0} & \forall \boldsymbol{s} \in \boldsymbol{T} \\ {[\mathcal{B}(\boldsymbol{t}), \underset{\sim}{\boldsymbol{\tau}}]+\left[\underset{\sim}{\boldsymbol{\tau}}, \mathcal{C}^{\prime}(\underset{\sim}{\boldsymbol{u}})\right]=0} & \forall \underset{\sim}{\boldsymbol{\tau}} \in \boldsymbol{\Sigma} \\ {[\mathcal{C}(\underset{\sim}{\boldsymbol{\sigma}}), \underset{\sim}{\boldsymbol{v}}]=[\mathcal{F}, \underset{\sim}{\boldsymbol{v}}]} & \underset{\sim}{\boldsymbol{v}} \in \boldsymbol{M}\end{cases}
$$

where, $[\mathcal{F}, \underset{\sim}{\boldsymbol{v}}]=(\boldsymbol{f}, \boldsymbol{v}), \forall \underset{\sim}{\boldsymbol{v}} \in \boldsymbol{M}$, and $\mathcal{B}^{\prime}$ and $\mathcal{C}^{\prime}$ denote the dual operators of $\boldsymbol{B}$ and $\mathcal{C}$, respectively. 


\section{Solvability of the continuous problem}

To prove existence, uniqueness and stability conditions of

$$
(\boldsymbol{t}, \underset{\sim}{\boldsymbol{\sigma}}, \underset{\sim}{\boldsymbol{u}})=(\boldsymbol{t},(\boldsymbol{\sigma}, p),(\boldsymbol{u}, \boldsymbol{\omega})) \in \boldsymbol{T} \times \boldsymbol{\Sigma} \times \boldsymbol{M}
$$

solution of (9), we shall recall some technical results given bellow. These results concern the properties of the operators $\mathcal{A}, \mathcal{B}$ and $\mathcal{C}$. Mainly:

- $\mathcal{A}$ is bounded, continuous and strictly monotone,

- $\mathcal{B}$ verifies the inf-sup condition on the Kernel of $\mathcal{C}$,

- $\mathcal{C}$ verifies the inf-sup condition.

Here we give several technical lemmas that establish the appropriate conditions on the operators $\mathcal{A}$. These properties are given for both Power and Carreau laws. In the following, to distinguish the two models, we set $\delta=0$ for Power law and $\delta=1$ for Carreau law. For details on the following three Lemmas, see Sandri [17].

Lemma 3.1. Let $\boldsymbol{s}, \boldsymbol{t} \in \boldsymbol{T}=\left[L^{r}(\Omega)\right]^{2 \times 2}$, we have the following results: For $1<r<2$,

$$
\begin{aligned}
{[\mathcal{A}(\boldsymbol{s})-\mathcal{A}(\boldsymbol{t}), \boldsymbol{s}-\boldsymbol{t}] } & \geq C \frac{\|\boldsymbol{s}-\boldsymbol{t}\|_{0, r, \Omega}^{2}}{\delta+\|\boldsymbol{s}\|_{0, r, \Omega}^{2-r}+\|\boldsymbol{t}\|_{0, r, \Omega}^{2-r}}, \\
\|\mathcal{A}(\boldsymbol{s})-\mathcal{A}(\boldsymbol{t})\|_{0, r^{\prime}, \Omega} & \leq C\left[\int_{\Omega}|\mathcal{A}(\boldsymbol{s})-\mathcal{A}(\boldsymbol{t})||\boldsymbol{s}-\boldsymbol{t}| d x\right]^{1 / r^{\prime}} .
\end{aligned}
$$

Lemma 3.2. Let $\boldsymbol{s}, \boldsymbol{t} \in \boldsymbol{T}=\left[L^{r}(\Omega)\right]^{2 \times 2}$, we have the following results: For $r \geq 2$,

$$
\begin{aligned}
{[\mathcal{A}(s)-\mathcal{A}(\boldsymbol{t}), \boldsymbol{s}-\boldsymbol{t}] } & \geq C\left(\|\boldsymbol{s}-\boldsymbol{t}\|_{0, r, \Omega}^{r}+\int_{\Omega}\left(\delta+|\boldsymbol{s}|^{r-2}+|\boldsymbol{t}|^{r-2}\right)|\boldsymbol{s}-\boldsymbol{t}|^{2} d x\right), \\
\|\mathcal{A}(\boldsymbol{s})-\mathcal{A}(\boldsymbol{t})\|_{0, r^{\prime}, \Omega} & \leq C\left[\int_{\Omega}\left(\delta+|\boldsymbol{s}|^{r-2}+|\boldsymbol{t}|^{r-2}\right)|\boldsymbol{s}-\boldsymbol{t}|^{2} d x\right]^{1 / 2}\left(\delta+\|\boldsymbol{s}\|_{0, r, \Omega}^{(r-2) / 2}+\|\boldsymbol{t}\|_{0, r, \Omega}^{(r-2) / 2}\right) .
\end{aligned}
$$

Lemma 3.3. Let $\boldsymbol{s}, \boldsymbol{t} \in \boldsymbol{T}=\left[L^{r}(\Omega)\right]^{2 \times 2}$, we have the following inequality: For $r>1$,

$$
[\mathcal{A}(s)-\mathcal{A}(\boldsymbol{t}), s-\boldsymbol{t}] \geq C \int_{\Omega}|\mathcal{A}(s)-\mathcal{A}(\boldsymbol{t})||s-\boldsymbol{t}| d x .
$$

Remark 3.1. The above Lemmas 3.1, 3.2 and 3.3, imply that the operator $\mathcal{A}$ is bounded, continuous and strictly monotone on reflexive Banach spaces.

We also state two known results that will be utilized.

Lemma 3.4. Let $\left(X,\|\cdot\|_{X}\right)$ and $\left(M,\|\cdot\|_{M}\right)$ be two reflexive Banach spaces. Let $\left(X^{\prime},\|\cdot\|_{X^{\prime}}\right)$ and $\left(M^{\prime},\|\cdot\|_{M^{\prime}}\right)$ be their corresponding dual space. Let $B: X \longrightarrow M^{\prime}$ be a linear continuous operator, and $B^{\prime}: M \longrightarrow X^{\prime}$ its dual. Let $V=\operatorname{Ker}(B)$ be the kernel of $B$; we denote by $V^{o} \subset X^{\prime}$ the polar set of $V$, i.e., $V^{o}=\left\{x^{\prime} \in X^{\prime},\left\langle x^{\prime}, v>=0, \forall v \in V\right\}\right.$, and $\dot{B}:(X / V) \longrightarrow M^{\prime}$ the quotient operator associated to $B$. Then, the three following properties are equivalent:

(i) there exists $\beta>0$, such that

$$
\inf _{q \in M} \sup _{v \in X} \frac{[B(v), q]}{\|q\|_{M}\|v\|_{X}} \geq \beta
$$

(ii) $B^{\prime}$ is an isomorphism from $M$ onto $V^{o}$ and

$$
\left\|B^{\prime} q\right\|_{X^{\prime}} \geq \beta\|q\|_{M} \quad \forall q \in M
$$

(iii) $\dot{B}$ is an isomorphism from $(X / V)$ onto $M^{\prime}$ and

$$
\|\dot{B} \dot{v}\|_{M^{\prime}} \geq \beta\|\dot{v}\|_{(X / V)} \quad \forall \dot{v} \in(X / V) .
$$


The proof of this Lemma is given in Sandri [17] (see also Remark 4.2, p. 61, in Girault and Raviart [13]).

Lemma 3.5. ([16], Theorem 9.45, p. 361, Browder-Minty). Let $X$ be a real reflexive Banach space and let $K: X \longrightarrow X^{\prime}$ be bounded, continuous, coercive and monotone. Then for any $g \in X^{\prime}$ there exists a solution $u$ of the equation $K(u)=g$; i.e. $K(X)=X^{\prime}$.

Now, we establish the appropriate inf-sup conditions for the operators $\mathcal{B}$ and $\mathcal{C}$. Define $\boldsymbol{Z}_{1}$, the null space for $\mathcal{C}$ :

$$
\boldsymbol{Z}_{1}=\{\underset{\sim}{\boldsymbol{\tau}} \in \boldsymbol{\Sigma} ;[\mathcal{C}(\underset{\sim}{\boldsymbol{\tau}}), \underset{\sim}{\boldsymbol{v}}]=0, \forall \underset{\sim}{\boldsymbol{v}} \in \boldsymbol{M}\}
$$

Lemma 3.6. There exists a positive constant $\beta_{1}$ such that

$$
\inf _{\underset{\sim}{\boldsymbol{\tau}} \in \boldsymbol{Z}_{1}} \sup _{\boldsymbol{s} \in \boldsymbol{T}} \frac{[\boldsymbol{\mathcal { B }}(\boldsymbol{s}), \underset{\sim}{\boldsymbol{\tau}}]}{\|\boldsymbol{s}\|_{T}\|\underset{\sim}{\boldsymbol{\tau}}\|_{\Sigma}} \geq \beta_{1}
$$

Proof. Let $\underset{\sim}{\boldsymbol{\tau}}=(\boldsymbol{\tau}, q) \in \boldsymbol{Z}_{1}$. Then, $\boldsymbol{\tau} \in\left[L^{r^{\prime}}(\Omega)\right]^{2 \times 2}, q \in L_{0}^{r^{\prime}}(\Omega)$ and $\boldsymbol{\operatorname { d i v }}(\boldsymbol{\tau}-q \boldsymbol{I})=0$. Now, let $\boldsymbol{s}^{*}=-|\boldsymbol{\tau}|^{r^{\prime}-2} \boldsymbol{\tau}$. We have $\boldsymbol{s}^{*} \in \boldsymbol{T}$ and $\left\|\boldsymbol{s}^{*}\right\|_{T}=\|\boldsymbol{\tau}\|_{0, r^{\prime}, \Omega}^{r^{\prime}-1}$. Thus,

$$
\frac{\left[\mathcal{B}\left(s^{*}\right), \underset{\sim}{\tau}\right]}{\left\|\boldsymbol{s}^{*}\right\|_{T}}=\frac{-\left(\boldsymbol{s}^{*}, \boldsymbol{\tau}\right)}{\left\|\boldsymbol{s}^{*}\right\|_{T}}=\frac{\|\boldsymbol{\tau}\|_{0, r^{\prime}, \Omega}^{r^{\prime}}}{\|\boldsymbol{\tau}\|_{0, r^{\prime}, \Omega}^{r^{\prime}-1}}=\|\boldsymbol{\tau}\|_{0, r^{\prime}, \Omega} .
$$

On the other hand, since $\boldsymbol{d i v}(\boldsymbol{\tau}-q \boldsymbol{I})=0$, there exists a constant $C>0$ such that (see Lemma 4 in [15]) $\|q\|_{0, r^{\prime}, \Omega} \leq C\|\boldsymbol{\tau}\|_{0, r^{\prime}, \Omega}$. Therefore, for all $\underset{\sim}{\boldsymbol{\tau}} \in \boldsymbol{Z}_{1}$, we have

$$
\sup _{\boldsymbol{s} \in \boldsymbol{T}} \frac{[\mathcal{B}(\boldsymbol{s}), \boldsymbol{\tau}]}{\|\boldsymbol{s}\|_{T}} \geq \frac{\left[\mathcal{B}\left(\boldsymbol{s}^{*}\right), \underset{\sim}{\boldsymbol{\tau}}\right]}{\left\|\boldsymbol{s}^{*}\right\|_{T}}=\|\boldsymbol{\tau}\|_{0, r^{\prime}, \Omega} \geq C\|\|_{\sim}^{\boldsymbol{\tau}} \|_{\Sigma}
$$

which completes the proof.

Lemma 3.7. There exists a positive constant $\beta_{2}$ such that

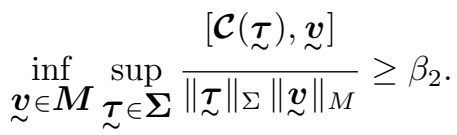

Proof. The proof of this result is the same as the one of Proposition 2.2 in [9].

The main result of this section can be established now.

Theorem 3.1. Problem (9) admits a unique solution $(\boldsymbol{t}, \underset{\sim}{\boldsymbol{\sigma}}, \underset{\sim}{\boldsymbol{u}}) \in \boldsymbol{T} \times \boldsymbol{\Sigma} \times \boldsymbol{M}$ satisfying the following stability condition,

$$
\|\boldsymbol{t}\|_{T}+\|\underset{\sim}{\boldsymbol{g}}\|_{\Sigma}+\|\underset{\sim}{\boldsymbol{u}}\|_{M} \leq C(\boldsymbol{f}),
$$

where $C(\boldsymbol{f})$ is a positive constant depending on $\boldsymbol{f}$.

Proof. Using the Inf-Sup condition (11) and applying Lemma 3.4(iii) to the operator $\mathcal{C}$, we get the existence of $\underset{\sim}{\boldsymbol{\sigma}}=(\dot{\boldsymbol{\sigma}}, \dot{p}) \in \boldsymbol{\Sigma} / \boldsymbol{Z}_{1}$ such that

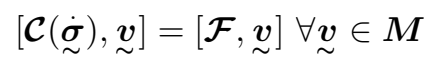


and

$$
\|\stackrel{\dot{\boldsymbol{\sigma}}}{\sim}\|_{\boldsymbol{\Sigma} / \boldsymbol{Z}_{1}} \leq C\|\mathbf{f}\|_{0, r^{\prime}, \Omega}
$$

Let $\underset{\sim}{\boldsymbol{\sigma}^{0}}=\left(\boldsymbol{\sigma}^{0}, p^{0}\right) \in \underset{\sim}{\dot{\boldsymbol{\sigma}}}$ such that

$$
\left\|\boldsymbol{\sigma}^{0}\right\|_{\boldsymbol{\Sigma}}=\|\underset{\sim}{\dot{\boldsymbol{\sigma}}}\|_{\boldsymbol{\Sigma} / \boldsymbol{Z}_{1}} \leq C\|\mathbf{f}\|_{0, r^{\prime}, \Omega}
$$


way: Find $\boldsymbol{t} \in \boldsymbol{T}$, and ${\underset{\sim}{\sim}}^{*} \in \boldsymbol{Z}_{1}$ such that

$$
\begin{cases}{[\mathcal{A}(\boldsymbol{t}), \boldsymbol{s}]+\left[\boldsymbol{s}, \mathcal{B}^{\prime}\left(\boldsymbol{\sigma}^{*}\right)\right]=-\left[\boldsymbol{s}, \mathcal{B}^{\prime}\left(\boldsymbol{\sigma}^{0}\right)\right]} & \forall \boldsymbol{s} \in \boldsymbol{T}, \\ {[\mathcal{B}(\boldsymbol{t}), \boldsymbol{\sim}]=0} & \forall \underset{\sim}{\boldsymbol{\tau}} \in \boldsymbol{Z}_{1}\end{cases}
$$

Now, from the properties of the operator $\mathcal{A}$ (see Remark 3.1) and Lemma 3.5, there exists a $\boldsymbol{t} \in \boldsymbol{Z}_{2}$ such that

$$
[\mathcal{A}(\boldsymbol{t}), s]=-\left[s, \mathcal{B}^{\prime}\left(\boldsymbol{\sigma}^{0}\right)\right] \forall s \in Z_{2},
$$

where $\boldsymbol{Z}_{2}=\operatorname{Ker}(\boldsymbol{B})=\{s \in \boldsymbol{T} ;[\boldsymbol{B}(s), \underset{\sim}{\boldsymbol{\tau}}]=0\}, \underset{\sim}{\forall \boldsymbol{\tau}} \in \boldsymbol{\Sigma}$. Uniqueness of $\boldsymbol{t}$ is implied by the fact that the operator $\mathcal{A}$ is strictly monotone (see Remark 3.1).

Furthermore, one can prove that

$$
\|\boldsymbol{t}\|_{0, r, \Omega} \leq C\left(\|\mathbf{f}\|_{0, r^{\prime}, \Omega}+\|\mathbf{f}\|_{0, r^{\prime}, \Omega}^{r^{\prime} / r}\right) .
$$

Equation (16) implies $\left[\boldsymbol{A}(\boldsymbol{t})+\mathcal{B}^{\prime}\left({\underset{\sim}{\boldsymbol{\sigma}}}^{0}\right), \boldsymbol{s}\right]=0 \forall \boldsymbol{s} \in \boldsymbol{Z}_{2}$, i.e, $\mathcal{A}(\boldsymbol{t})+\mathcal{B}^{\prime}\left({\underset{\sim}{\boldsymbol{\sigma}}}^{0}\right) \in \boldsymbol{Z}_{2}^{o}$. Thus, by the Inf-Sup condition (10) and Lemma 3.4(ii), there exists a unique $\boldsymbol{\sigma}^{*} \in \boldsymbol{Z}_{1}$ such that

$$
\left[\mathcal{B}^{\prime}\left(\underline{\sim}^{*}\right), s\right]=-\left[\mathcal{A}(\boldsymbol{t})+\mathcal{B}^{\prime}\left(\underline{\sim}^{0}\right), s\right] \forall s \in \boldsymbol{T}
$$

and

$$
\left\|\boldsymbol{\sigma}^{*}\right\|_{\boldsymbol{\Sigma}} \leq C\left\|\boldsymbol{A}(\boldsymbol{t})+\mathcal{B}^{\prime}\left({\underset{\sim}{0}}^{0}\right)\right\|_{0, r^{\prime}, \Omega}
$$

Therefore, from (12) and (18), we get

$$
[\mathcal{A}(\boldsymbol{t}), \boldsymbol{s}]+\left[\boldsymbol{s}, \boldsymbol{\mathcal { B }}^{\prime}(\underset{\sim}{\boldsymbol{\sigma}})\right]=0 \forall \boldsymbol{s} \in \boldsymbol{T}, \quad[\mathcal{C}(\underset{\sim}{\boldsymbol{\sigma}}), \underset{\sim}{\boldsymbol{v}}]=[\mathcal{F}, \underset{\sim}{\boldsymbol{v}}] \underset{\sim}{\forall} \in \boldsymbol{M}
$$

and, owing to (14), (17) and (19), we have

$$
\|\boldsymbol{t}\|_{\boldsymbol{T}}+\|\underset{\sim}{\boldsymbol{\sigma}}\|_{\boldsymbol{\Sigma}} \leq C(\boldsymbol{f})
$$

where $C(\boldsymbol{f})$ is a positive constant depending on $\boldsymbol{f}$.

On the other hand, since $[\boldsymbol{B}(\boldsymbol{t}), \boldsymbol{\tau}]=0 \underset{\sim}{\forall \boldsymbol{\tau}} \in \boldsymbol{Z}_{1}$, we have $\boldsymbol{B}(\boldsymbol{t}) \in \boldsymbol{Z}_{1}^{o}$. Thus, by the Inf-Sup condition (11) and Lemma 3.4(ii), there exists a unique $\underset{\sim}{\boldsymbol{u}} \in \boldsymbol{M}$ such that $\left[\underset{\sim}{\boldsymbol{\tau}}, \mathcal{C}^{\prime}(\underset{\sim}{\boldsymbol{u}})\right]=-[\boldsymbol{B}(\boldsymbol{t}), \underset{\sim}{\boldsymbol{\tau}}] \underset{\sim}{\forall \boldsymbol{\tau}} \in \boldsymbol{\Sigma}$ and $\|\underset{\sim}{\boldsymbol{u}}\|_{\boldsymbol{M}} \leq C\|\boldsymbol{B}(\boldsymbol{t})\|_{\boldsymbol{\Sigma}^{\prime}}$. Therefore, $[\boldsymbol{B}(\boldsymbol{t}), \underset{\sim}{\boldsymbol{\tau}}]+\left[\underset{\sim}{\boldsymbol{\tau}}, \mathcal{C}^{\prime}(\underset{\sim}{\boldsymbol{u}})\right]=0 \underset{\sim}{\forall \boldsymbol{\tau}} \in \boldsymbol{\Sigma}$ and $\|\underset{\sim}{\boldsymbol{u}}\|_{\boldsymbol{M}} \leq C(\boldsymbol{f})$, which completes the proof. 


\section{Discrete mixed formulation and error analysis}

We assume that the boundary $\Gamma$ of the domain $\Omega$ is polygonal. We first give some finite element notations. Let $h>0$ and $\mathcal{T}_{h}$ a triangulation of $\Omega$ into triangles. We assume that the triangulation $\mathcal{T}_{h}$ is regular in the sense of Ciarlet [5]. Let $K \in \mathcal{T}_{h}$, be an element of the triangulation, we denote by $b_{K}$ the bubble function defined by:

$$
b_{K}(x)=\lambda_{1}(x) \lambda_{2}(x) \lambda_{3}(x), \forall x \in K,
$$

$\lambda_{i}, i=1, \cdots, 3$ being the barycentric co-ordinates with respect to the element $K$. Let $P_{k}(K)$ denote the space of polynomials of degree less than or equal to $k$ on $K$, and

$$
R(K)=\left[P_{1}(K)\right]^{2} \oplus \mathbb{R} \operatorname{curl} b_{K},
$$

where $\operatorname{curl} b_{K}=\left(\frac{\partial b_{K}}{\partial x_{2}},-\frac{\partial b_{K}}{\partial x_{1}}\right)$.

To write the discrete mixed formulation, we introduce the following finite dimensional spaces:

$$
\begin{aligned}
\boldsymbol{T}_{h} & =\left\{\boldsymbol{s}_{h} \in \boldsymbol{T} ; \boldsymbol{s}_{\left.h\right|_{K}} \in R(K), \forall K \in \mathcal{T}_{h}\right\} \\
\boldsymbol{\Sigma}_{h} & =\left\{\underset{\sim}{\boldsymbol{\tau}_{\boldsymbol{h}}}=\left(\boldsymbol{\tau}_{h}, q_{h}\right) \in \boldsymbol{\Sigma} ; \boldsymbol{\tau}_{\left.h\right|_{K}} \in[R(K)]^{2}, q_{\left.\right|_{K}} \in P_{1}(K), \forall K \in \mathcal{T}_{h}\right\}, \\
\boldsymbol{M}_{h} & =\left\{\underset{\sim}{\boldsymbol{v}_{\boldsymbol{h}}}=\left(\boldsymbol{v}_{h}, \boldsymbol{\eta}_{h}\right) \in \boldsymbol{M} ; \boldsymbol{v}_{\left.\right|_{K}} \in\left[P_{0}(K)\right]^{2}, \boldsymbol{\eta}_{h}=\theta_{h} \boldsymbol{\chi}, \theta_{\left.h\right|_{K}} \in P_{1}(K), \forall K \in \mathcal{T}_{h}\right\},
\end{aligned}
$$

where

$$
\chi=\left[\begin{array}{rr}
0 & -1 \\
1 & 0
\end{array}\right]
$$

The discrete mixed formulation of problem (9) is given by: Find $\boldsymbol{t}_{h} \in \boldsymbol{T}_{h}, \underset{\sim}{\boldsymbol{\sigma}_{\boldsymbol{h}}} \in \boldsymbol{\Sigma}_{h}$ and $\underset{\sim}{\boldsymbol{u}_{\boldsymbol{h}}} \in \boldsymbol{M}_{h}$ such that

$$
\begin{cases}{\left[\mathcal{A}\left(\boldsymbol{t}_{h}\right), \boldsymbol{s}_{h}\right]+\left[\boldsymbol{s}_{h}, \mathcal{B}^{\prime}\left(\boldsymbol{\sigma}_{\boldsymbol{h}}\right)\right]=0} & \forall \boldsymbol{s}_{h} \in \boldsymbol{T}_{h}, \\ {\left[\mathcal{B}\left(\boldsymbol{t}_{h}\right), \boldsymbol{\tau}_{\boldsymbol{\sim}}\right]+\left[\boldsymbol{\tau}_{\boldsymbol{\sim}}, \mathcal{C}^{\prime}\left(\boldsymbol{u}_{\boldsymbol{h}}\right)\right]=0} & \forall \boldsymbol{\tau}_{\boldsymbol{\sim}} \in \boldsymbol{\Sigma}_{h}, \\ {\left[\mathcal{C}\left(\boldsymbol{\sigma}_{\boldsymbol{\sim}}\right),{\underset{\sim}{\boldsymbol{h}}}_{\boldsymbol{\sim}}\right]=\left[\mathcal{F}, \boldsymbol{v}_{\boldsymbol{\sim}}^{\boldsymbol{v}_{\boldsymbol{h}}}\right]} & \forall \underset{\sim}{\boldsymbol{v}_{\boldsymbol{h}}} \in \boldsymbol{M}_{h} .\end{cases}
$$

In order to analyze the discrete problem (20) and prove error estimates, we have to state some intermediate results.

Lemma 4.1. There exists a positive constant $\beta_{1}^{*}$ independent of $h$, such that

$$
\inf _{\boldsymbol{\tau}_{\sim} \in \boldsymbol{Z}_{1}^{h}} \sup _{\boldsymbol{s}_{h} \in \boldsymbol{T}_{h}} \frac{\left[\mathcal{B}\left(\boldsymbol{s}_{h}\right), \boldsymbol{\tau}_{\boldsymbol{\sim}}\right]}{\left\|\boldsymbol{s}_{h}\right\|_{T}\left\|\boldsymbol{\tau}_{\boldsymbol{h}}\right\|_{\Sigma}} \geq \beta_{1}^{*},
$$

where $\boldsymbol{Z}_{1}^{h}$ is the discrete Kernel of $\mathcal{C}$, i.e.,

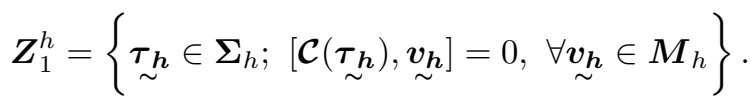

Proof. Let $\underset{\sim}{\boldsymbol{\tau}_{h}}=\left(\boldsymbol{\tau}_{h}, q_{h}\right) \in \boldsymbol{Z}_{1}^{h}$. Then, $\boldsymbol{\tau}_{h} \in\left[L^{r^{\prime}}(\Omega)\right]^{2 \times 2}, q_{h} \in L_{0}^{r^{\prime}}(\Omega)$ and $\boldsymbol{d i v}\left(\boldsymbol{\tau}_{h}-q_{h} \boldsymbol{I}\right)=0$. Now, let $\boldsymbol{s}^{*}=-\left|\boldsymbol{\tau}_{h}\right|^{r^{\prime}-2} \boldsymbol{\tau}_{h}$ and $\boldsymbol{s}_{h}^{*} \in \boldsymbol{T}_{h}$ such that

$$
\int_{\Omega} \boldsymbol{s}_{h}^{*} \phi_{h} d x=\int_{\Omega} \boldsymbol{s}^{*} \phi_{h} d x, \forall \phi_{h} \in \boldsymbol{T}_{h}
$$


Proceeding as for the proof of Lemma 4.1 in [6], we have $\left\|s_{h}^{*}\right\|_{T^{\prime}} \leq C\left\|\boldsymbol{s}^{*}\right\|_{T}$, where $C$ is a positive constant independent of $h$. Thus, $\boldsymbol{s}_{h}^{*} \in \boldsymbol{T}_{h}$ and $\left\|\boldsymbol{s}_{h}^{*}\right\|_{T} \leq C\left\|\boldsymbol{\tau}_{h}\right\|_{0, r^{\prime}, \Omega}^{r^{\prime}-1}$. Therefore,

$$
\frac{\left[\mathcal{B}\left(s_{h}^{*}\right), \boldsymbol{\tau}_{\sim}\right]}{\left\|s_{h}^{*}\right\|_{T}}=\frac{-\left(s_{h}^{*}, \boldsymbol{\tau}_{h}\right)}{\left\|s_{h}^{*}\right\|_{T}} \geq C \frac{\left\|\boldsymbol{\tau}_{h}\right\|_{0, r^{\prime}, \Omega}^{r^{\prime}}}{\left\|\boldsymbol{\tau}_{h}\right\|_{0, r^{\prime}, \Omega}^{r^{\prime}-1}}=C\left\|\boldsymbol{\tau}_{h}\right\|_{0, r^{\prime}, \Omega} .
$$

On the other hand, since $\boldsymbol{d i v}\left(\boldsymbol{\tau}_{h}-q_{h} \boldsymbol{I}\right)=0$, there exists a constant $C>0$ such that (see Lemma 4.3 below) $\left\|q_{h}\right\|_{0, r^{\prime}, \Omega} \leq C\left\|\boldsymbol{\tau}_{h}\right\|_{0, r^{\prime}, \Omega}$. Therefore, for all $\boldsymbol{\tau}_{\boldsymbol{\sim}} \in \boldsymbol{Z}_{1}^{h}$, we have

$$
\sup _{\boldsymbol{s}_{h} \in \boldsymbol{T}_{h}} \frac{\left[\mathcal{B}\left(\boldsymbol{s}_{h}\right), \boldsymbol{\tau}_{\boldsymbol{h}}\right]}{\left\|\boldsymbol{s}_{h}\right\|_{T}} \geq \frac{\left[\mathcal{B}\left(\boldsymbol{s}_{h}^{*}\right), \boldsymbol{\tau}_{\boldsymbol{\sim}}\right]}{\left\|\boldsymbol{s}_{h}^{*}\right\|_{T}} \geq C\left\|\boldsymbol{\tau}_{\sim}\right\|_{\Sigma}
$$

which completes the proof.

For the proofs of the two following results, see Farhloul and Zine [9].

Lemma 4.2. There exists a positive constant $\beta_{2}^{*}$ independent of $h$, such that

$$
\inf _{\boldsymbol{v}_{\boldsymbol{h}} \in \boldsymbol{M}_{h}} \sup _{\boldsymbol{\tau}_{\boldsymbol{\sim}} \in \boldsymbol{\Sigma}_{h}} \frac{\left[\mathcal{C}\left(\boldsymbol{\tau}_{\boldsymbol{h}}\right), \boldsymbol{v}_{\boldsymbol{\sim}}\right]}{\left\|\boldsymbol{\tau}_{\boldsymbol{\sim}}\right\|_{\Sigma}\left\|\boldsymbol{v}_{\boldsymbol{\sim}}\right\|_{M}} \geq \beta_{2}^{*}
$$

Lemma 4.3. There exists a positive constant $C$, independent of $h$, such that

$$
\forall \boldsymbol{\tau}_{\boldsymbol{\sim}} \in \boldsymbol{Z}_{1}^{h}, \quad\left\|q_{h}\right\|_{0, r^{\prime}, \Omega} \leq C\left\|\boldsymbol{\tau}_{h}\right\|_{0, r^{\prime}, \Omega}
$$

Due to the previous Lemma, the discrete inf-sup conditions (21) and (22), we obtain, as for the continuous problem, the existence, uniqueness and stability of the discrete solution. More precisely,

Theorem 4.1. Problem (20) admits a unique solution $\left(\boldsymbol{t}_{h}, \boldsymbol{\sigma}_{\boldsymbol{h}}, \boldsymbol{u}_{\boldsymbol{h}}\right) \in \boldsymbol{T}_{h} \times \boldsymbol{\Sigma}_{h} \times \boldsymbol{M}_{h}$ satisfying the following stability condition,

$$
\left\|\boldsymbol{t}_{h}\right\|_{T}+\left\|\underset{\sim}{\boldsymbol{\sigma}_{h}}\right\|_{\Sigma}+\left\|\underset{\sim}{\boldsymbol{u}_{\boldsymbol{h}}}\right\|_{M} \leq C(\boldsymbol{f}) .
$$

wher $C(\boldsymbol{f})$ is a positive constant depending on $\boldsymbol{f}$ and independent of $h$.

To prove the error estimates, we shall use the following result (see Farhloul and Zine [9]).

Proposition 4.1. Let $s$ be a real such that $s>1$, then there exists an operator

$$
\begin{aligned}
\Pi_{h}: & {\left[W^{1, s}(\Omega)\right]^{2 \times 2} \times\left(W^{1, s}(\Omega) \cap L_{0}^{s}(\Omega)\right) \longrightarrow \boldsymbol{\Sigma}_{h} } \\
& \underset{\sim}{\boldsymbol{\tau}} \longmapsto \Pi_{h}(\underset{\sim}{\boldsymbol{\tau}})
\end{aligned}
$$

which satisfies

$$
\left[\mathcal{C}\left(\underset{\sim}{\boldsymbol{\tau}}-\Pi_{h}(\underset{\sim}{\boldsymbol{\tau}}),{\underset{\sim}{\boldsymbol{v}}}_{\boldsymbol{\sim}}\right]=0, \forall \underset{\sim}{\boldsymbol{v}_{\boldsymbol{h}}} \in M_{h}\right.
$$

Further, for all $\underset{\sim}{\boldsymbol{\tau}}=(\boldsymbol{\tau}, q) \in\left[W^{m, s}(\Omega)\right]^{2 \times 2} \times\left(W^{m, s}(\Omega) \cap L_{0}^{s}(\Omega)\right), m=1,2$, we have

$$
\left\|(\boldsymbol{\tau}, q)-\Pi_{h}(\boldsymbol{\tau}, q)\right\|_{0, s, \Omega} \leq C h^{m}\left(|\boldsymbol{\tau}|_{m, s, \Omega}+|q|_{m, s, \Omega}\right)
$$

where $C$ is a positive constant independent of $h$, and

$$
\|(\boldsymbol{\tau}, q)\|_{0, s, \Omega}=\left(\|\boldsymbol{\tau}\|_{0, s, \Omega}^{s}+\|q\|_{0, s, \Omega}^{s}\right)^{\frac{1}{s}}
$$


We are now able to state the error estimates for problem (20). In the following

$$
(\boldsymbol{t}, \underset{\sim}{\boldsymbol{\sigma}}, \underset{\sim}{\boldsymbol{u}})=(\boldsymbol{t},(\boldsymbol{\sigma}, p),(\boldsymbol{u}, \boldsymbol{\omega})) \text { and }\left(\boldsymbol{t}_{h}, \underset{\sim}{\boldsymbol{\sigma}_{h}}, \underset{\sim}{\boldsymbol{u}_{h}}\right)=\left(\boldsymbol{t}_{h},\left(\boldsymbol{\sigma}_{h}, p_{h}\right),\left(\boldsymbol{u}_{h}, \boldsymbol{\omega}_{h}\right)\right)
$$

denote respectively the solution of the continuous problem (9) and the solution of the discrete problem (20). In the sequel, we use the following notation:

$$
\boldsymbol{u}_{h}^{*}=P_{h}^{0} \boldsymbol{u}, \text { the } L^{2} \text {-projection of } \boldsymbol{u} \text { onto }\left[\prod_{K \in \mathcal{T}_{h}} P_{0}(K)\right]^{2} .
$$

Theorem 4.2. Let $1<r<2$ and $m=1$, 2. Suppose $\boldsymbol{t} \in\left[W^{m, r}(\Omega)\right]^{2 \times 2}, \underset{\sim}{\boldsymbol{\sigma}}=(\boldsymbol{\sigma}, p) \in\left[W^{m, r^{\prime}}(\Omega)\right]^{2 \times 2} \times$ $W^{m, r^{\prime}}(\Omega)$, and $\underset{\sim}{\boldsymbol{u}}=(\boldsymbol{u}, \boldsymbol{\omega}) \in\left[W^{1, r}(\Omega)\right]^{2} \times\left[W^{m, r}(\Omega)\right]^{2 \times 2}$. Then, there exists a positive constant $C$ independent of $h$ such that

$$
\begin{aligned}
&\left\|\boldsymbol{t}-\boldsymbol{t}_{h}\right\|_{0, r, \Omega} \leq C h^{m(r / 2)}, \\
&\left\|\boldsymbol{\sigma}-\boldsymbol{\sigma}_{h}\right\|_{0, r^{\prime}, \Omega}+\left\|p-p_{h}\right\|_{0, r^{\prime}, \Omega} \leq C h^{m(r-1)}, \\
&\left\|\boldsymbol{u}_{h}^{*}-\boldsymbol{u}_{h}\right\|_{0, r, \Omega}+\left\|\boldsymbol{\omega}-\boldsymbol{\omega}_{h}\right\|_{0, r, \Omega} \leq C h^{m(r / 2)}, \\
&\left\|\boldsymbol{u}-\boldsymbol{u}_{h}\right\|_{0, r, \Omega} \leq C h^{r / 2} \quad \text { if } m=1, \quad \text { and } \quad\left\|\boldsymbol{u}-\boldsymbol{u}_{h}\right\|_{0, r, \Omega} \leq C h \quad \text { if } m=2 .
\end{aligned}
$$

Proof. By subtracting the last equation of (20) from the last equation of (9), we get for $\underset{\sim}{\boldsymbol{v}}=(\boldsymbol{v}, \boldsymbol{\eta})=$ $\left(\boldsymbol{v}_{h}, \boldsymbol{\eta}_{h}\right)$

$$
\left[\mathcal{C}\left(\underset{\sim}{\boldsymbol{\sigma}}-\boldsymbol{\sigma}_{\boldsymbol{\sim}}\right), \underset{\sim}{\boldsymbol{v}_{\boldsymbol{h}}}\right)=0
$$

and then, owing to (24),

$$
\left(\boldsymbol{d i v}\left[\left(\boldsymbol{\sigma}_{h}^{*}-p_{h}^{*} \boldsymbol{I}\right)-\left(\boldsymbol{\sigma}_{h}-p_{h} \boldsymbol{I}\right)\right], \boldsymbol{v}_{h}\right)+\left(\boldsymbol{\sigma}_{h}^{*}-\boldsymbol{\sigma}_{h}, \boldsymbol{\eta}_{h}\right)=0,
$$

where $\left(\boldsymbol{\sigma}_{h}^{*}, p_{h}^{*}\right)=\Pi_{h}(\boldsymbol{\sigma}, p)$.

Let $\left(\boldsymbol{u}_{h}^{*}, \boldsymbol{\omega}_{h}^{*}\right)=\left(P_{h}^{0} \boldsymbol{u}, P_{h}^{1} \boldsymbol{\omega}\right)$ denote the $L^{2}$-projection of $(\boldsymbol{u}, \boldsymbol{\omega})$ onto the space $\boldsymbol{M}_{h}$ and $\boldsymbol{t}_{h}^{*}=P_{h}^{*} \boldsymbol{t}$ the $L^{2}$-projection of $\boldsymbol{t}$ onto the space $\boldsymbol{T}_{h}$. By subtracting the second equation of (20) from the second equation of (9), we get for $\underset{\sim}{\boldsymbol{\tau}}=(\boldsymbol{\tau}, q)=\left(\boldsymbol{\tau}_{h}, q_{h}\right)$

$$
\left(\boldsymbol{t}-\boldsymbol{t}_{h}, \boldsymbol{\tau}_{h}\right)+\left(\boldsymbol{d i v}\left(\boldsymbol{\tau}_{h}-q_{h} \boldsymbol{I}\right), \boldsymbol{u}-\boldsymbol{u}_{h}\right)+\left(\boldsymbol{\tau}_{h}, \boldsymbol{\omega}-\boldsymbol{\omega}_{h}\right)=0,
$$

and then

$$
\left(\boldsymbol{t}-\boldsymbol{t}_{h}, \boldsymbol{\tau}_{h}\right)+\left(\boldsymbol{d i v}\left(\boldsymbol{\tau}_{h}-q_{h} \boldsymbol{I}\right), \boldsymbol{u}_{h}^{*}-\boldsymbol{u}_{h}\right)+\left(\boldsymbol{\tau}_{h}, \boldsymbol{\omega}_{h}^{*}-\boldsymbol{\omega}_{h}\right)=\left(\boldsymbol{\tau}_{h}, \boldsymbol{\omega}_{h}^{*}-\boldsymbol{\omega}\right) .
$$

This last relation and (30) yield

$$
\left(\boldsymbol{t}-\boldsymbol{t}_{h}, \boldsymbol{\sigma}_{h}^{*}-\boldsymbol{\sigma}_{h}\right)=\left(\boldsymbol{\sigma}_{h}^{*}-\boldsymbol{\sigma}_{h}, \boldsymbol{\omega}_{h}^{*}-\boldsymbol{\omega}\right)
$$

and then

$$
\left(\boldsymbol{t}_{h}^{*}-\boldsymbol{t}_{h}, \boldsymbol{\sigma}_{h}^{*}-\boldsymbol{\sigma}_{h}\right)=\left(\boldsymbol{\sigma}_{h}^{*}-\boldsymbol{\sigma}_{h}, \boldsymbol{\omega}_{h}^{*}-\boldsymbol{\omega}\right) .
$$

Now, by subtracting the first equation of (20) from the first equation of (9), we get for $s=s_{h}$

$$
\left(\mathcal{A}(\boldsymbol{t})-\mathcal{A}\left(\boldsymbol{t}_{h}\right), s_{h}\right)=\left(s_{h}, \boldsymbol{\sigma}-\boldsymbol{\sigma}_{h}\right) .
$$


Thus, using this last equation and (32), we have

$$
\begin{aligned}
\left(\mathcal{A}(\boldsymbol{t})-\mathcal{A}\left(\boldsymbol{t}_{h}\right), \boldsymbol{t}-\boldsymbol{t}_{h}\right)= & \left(\mathcal{A}(\boldsymbol{t})-\mathcal{A}\left(\boldsymbol{t}_{h}\right), \boldsymbol{t}-\boldsymbol{t}_{h}^{*}\right)+\left(\mathcal{A}(\boldsymbol{t})-\mathcal{A}\left(\boldsymbol{t}_{h}\right), \boldsymbol{t}_{h}^{*}-\boldsymbol{t}_{h}\right) \\
= & \left(\mathcal{A}(\boldsymbol{t})-\mathcal{A}\left(\boldsymbol{t}_{h}\right), \boldsymbol{t}-\boldsymbol{t}_{h}^{*}\right)+\left(\boldsymbol{t}_{h}^{*}-\boldsymbol{t}_{h}, \boldsymbol{\sigma}-\boldsymbol{\sigma}_{h}\right) \\
= & \left(\mathcal{A}(\boldsymbol{t})-\mathcal{A}\left(\boldsymbol{t}_{h}\right), \boldsymbol{t}-\boldsymbol{t}_{h}^{*}\right)+\left(\boldsymbol{t}_{h}^{*}-\boldsymbol{t}_{h}, \boldsymbol{\sigma}-\boldsymbol{\sigma}_{h}^{*}\right)+\left(\boldsymbol{t}_{h}^{*}-\boldsymbol{t}_{h}, \boldsymbol{\sigma}_{h}^{*}-\boldsymbol{\sigma}_{h}\right) \\
= & \left(\mathcal{A}(\boldsymbol{t})-\mathcal{A}\left(\boldsymbol{t}_{h}\right), \boldsymbol{t}-\boldsymbol{t}_{h}^{*}\right)+\left(\boldsymbol{t}_{h}^{*}-\boldsymbol{t}_{h}, \boldsymbol{\sigma}-\boldsymbol{\sigma}_{h}^{*}\right)+\left(\boldsymbol{\sigma}_{h}^{*}-\boldsymbol{\sigma}_{h}, \boldsymbol{\omega}_{h}^{*}-\boldsymbol{\omega}\right) \\
= & \left(\mathcal{A}(\boldsymbol{t})-\mathcal{A}\left(\boldsymbol{t}_{h}\right), \boldsymbol{t}-\boldsymbol{t}_{h}^{*}\right)+\left(\boldsymbol{t}_{h}^{*}-\boldsymbol{t}, \boldsymbol{\sigma}-\boldsymbol{\sigma}_{h}^{*}\right)+\left(\boldsymbol{t}-\boldsymbol{t}_{h}, \boldsymbol{\sigma}-\boldsymbol{\sigma}_{h}^{*}\right) \\
& +\left(\boldsymbol{\sigma}_{h}^{*}-\boldsymbol{\sigma}_{h}, \boldsymbol{\omega}_{h}^{*}-\boldsymbol{\omega}\right),
\end{aligned}
$$

therefore

$$
\begin{aligned}
\left(\mathcal{A}(\boldsymbol{t})-\mathcal{A}\left(\boldsymbol{t}_{h}\right), \boldsymbol{t}-\boldsymbol{t}_{h}\right)= & \left(\mathcal{A}(\boldsymbol{t})-\mathcal{A}\left(\boldsymbol{t}_{h}\right), \boldsymbol{t}-\boldsymbol{t}_{h}^{*}\right)+\left(\boldsymbol{t}_{h}^{*}-\boldsymbol{t}, \boldsymbol{\sigma}-\boldsymbol{\sigma}_{h}^{*}\right)+\left(\boldsymbol{t}-\boldsymbol{t}_{h}, \boldsymbol{\sigma}-\boldsymbol{\sigma}_{h}^{*}\right) \\
& +\left(\boldsymbol{\sigma}_{h}^{*}-\boldsymbol{\sigma}_{h}, \boldsymbol{\omega}_{h}^{*}-\boldsymbol{\omega}\right) .
\end{aligned}
$$

On the other hand, owing to (33), we have

$$
\left(\mathcal{A}(\boldsymbol{t})-\mathcal{A}\left(\boldsymbol{t}_{h}\right), \boldsymbol{s}_{h}\right)=\left(\boldsymbol{s}_{h}, \boldsymbol{\sigma}-\boldsymbol{\sigma}_{h}^{*}\right)+\left(\boldsymbol{s}_{h}, \boldsymbol{\sigma}_{h}^{*}-\boldsymbol{\sigma}_{h}\right) \forall \boldsymbol{s}_{h} \in \boldsymbol{T}_{h}
$$

and then

$$
\left(s_{h}, \boldsymbol{\sigma}_{h}^{*}-\boldsymbol{\sigma}_{h}\right)=\left(\mathcal{A}(\boldsymbol{t})-\mathcal{A}\left(\boldsymbol{t}_{h}\right), \boldsymbol{s}_{h}\right)+\left(\boldsymbol{s}_{h}, \boldsymbol{\sigma}_{h}^{*}-\boldsymbol{\sigma}\right) \forall s_{h} \in \boldsymbol{T}_{h} .
$$

Thus, from (30) and the discrete Inf-Sup condition (21), we have

$$
\beta_{1}^{*}\left\|\boldsymbol{\sigma}_{h}^{*}-\boldsymbol{\sigma}_{h}\right\|_{0, r^{\prime}} \leq\left\|\mathcal{A}(\boldsymbol{t})-\mathcal{A}\left(\boldsymbol{t}_{h}\right)\right\|_{0, r^{\prime}}+\left\|\boldsymbol{\sigma}-\boldsymbol{\sigma}_{h}^{*}\right\|_{0, r^{\prime}} .
$$

Using this last estimate and (34), we get

$$
\begin{aligned}
\left(\mathcal{A}(\boldsymbol{t})-\mathcal{A}\left(\boldsymbol{t}_{h}\right), \boldsymbol{t}-\boldsymbol{t}_{h}\right) & \leq\left\|\mathcal{A}(\boldsymbol{t})-\mathcal{A}\left(\boldsymbol{t}_{h}\right)\right\|_{0, r^{\prime}}\left\|\boldsymbol{t}-\boldsymbol{t}_{h}^{*}\right\|_{0, r} \\
& +\left\|\boldsymbol{t}-\boldsymbol{t}_{h}^{*}\right\|_{0, r}\left\|\boldsymbol{\sigma}-\boldsymbol{\sigma}_{h}^{*}\right\|_{0, r^{\prime}}+\left\|\boldsymbol{t}-\boldsymbol{t}_{h}\right\|_{0, r}\left\|\boldsymbol{\sigma}-\boldsymbol{\sigma}_{h}^{*}\right\|_{0, r^{\prime}} \\
& +C\left(\left\|\mathcal{A}(\boldsymbol{t})-\mathcal{A}\left(\boldsymbol{t}_{h}\right)\right\|_{0, r^{\prime}}+\left\|\boldsymbol{\sigma}-\boldsymbol{\sigma}_{h}^{*}\right\|_{0, r^{\prime}}\right)\left\|\boldsymbol{\omega}-\boldsymbol{\omega}_{h}^{*}\right\|_{0, r}
\end{aligned}
$$

thus

$$
\begin{aligned}
\left(\mathcal{A}(\boldsymbol{t})-\mathcal{A}\left(\boldsymbol{t}_{h}\right), \boldsymbol{t}-\boldsymbol{t}_{h}\right) & \leq\left\|\mathcal{A}(\boldsymbol{t})-\mathcal{A}\left(\boldsymbol{t}_{h}\right)\right\|_{0, r^{\prime}}\left\|\boldsymbol{t}-\boldsymbol{t}_{h}^{*}\right\|_{0, r} \\
& +C\left\|\mathcal{A}(\boldsymbol{t})-\mathcal{A}\left(\boldsymbol{t}_{h}\right)\right\|_{0, r^{\prime}}\left\|\boldsymbol{\omega}-\boldsymbol{\omega}_{h}^{*}\right\|_{0, r}+\left\|\boldsymbol{t}-\boldsymbol{t}_{h}\right\|_{0, r}\left\|\boldsymbol{\sigma}-\boldsymbol{\sigma}_{h}^{*}\right\|_{0, r^{\prime}} \\
& +\left\|\boldsymbol{t}-\boldsymbol{t}_{h}^{*}\right\|_{0, r}\left\|\boldsymbol{\sigma}-\boldsymbol{\sigma}_{h}^{*}\right\|_{0, r^{\prime}}+C\left\|\boldsymbol{\sigma}-\boldsymbol{\sigma}_{h}^{*}\right\|_{0, r^{\prime}}\left\|\boldsymbol{\omega}-\boldsymbol{\omega}_{h}^{*}\right\|_{0, r}
\end{aligned}
$$

Now, owing to Lemma 3.1, Lemma 3.3 and (36), we have

$$
\begin{aligned}
\frac{\left\|\boldsymbol{t}-\boldsymbol{t}_{h}\right\|_{0, r}^{2}}{\delta+\|\boldsymbol{t}\|_{0, r}^{2-r}+\left\|\boldsymbol{t}_{h}\right\|_{0, r}^{2-r}} & +\int_{\Omega}\left|\mathcal{A}(\boldsymbol{t})-\mathcal{A}\left(\boldsymbol{t}_{h}\right)\right|\left|\boldsymbol{t}-\boldsymbol{t}_{h}\right| d x \\
& \leq C\left[\int_{\Omega}\left|\mathcal{A}(\boldsymbol{t})-\mathcal{A}\left(\boldsymbol{t}_{h}\right)\right|\left|\boldsymbol{t}-\boldsymbol{t}_{h}\right| d x\right]^{1 / r^{\prime}}\left\|\boldsymbol{t}-\boldsymbol{t}_{h}^{*}\right\|_{0, r} \\
& +C\left[\int_{\Omega}\left|\mathcal{A}(\boldsymbol{t})-\mathcal{A}\left(\boldsymbol{t}_{h}\right)\right|\left|\boldsymbol{t}-\boldsymbol{t}_{h}\right| d x\right]^{1 / r^{\prime}}\left\|\boldsymbol{\omega}-\boldsymbol{\omega}_{h}^{*}\right\|_{0, r} \\
& +C\left\|\boldsymbol{t}-\boldsymbol{t}_{h}\right\|_{0, r}\left\|\boldsymbol{\sigma}-\boldsymbol{\sigma}_{h}^{*}\right\|_{0, r^{\prime}}+C\left\|\boldsymbol{t}-\boldsymbol{t}_{h}^{*}\right\|_{0, r}\left\|\boldsymbol{\sigma}-\boldsymbol{\sigma}_{h}^{*}\right\|_{0, r^{\prime}} \\
& +C\left\|\boldsymbol{\sigma}-\boldsymbol{\sigma}_{h}^{*}\right\|_{0, r^{\prime}}\left\|\boldsymbol{\omega}-\boldsymbol{\omega}_{h}^{*}\right\|_{0, r} .
\end{aligned}
$$

Then, using the Young inequality,

$$
a b \leq \frac{1}{r}(\varepsilon a)^{r}+\frac{1}{r^{\prime}}\left(\frac{b}{\varepsilon}\right)^{r^{\prime}}, \text { for } \varepsilon>0, a \geq 0 \text { and } b \geq 0,
$$


we have

$$
\begin{aligned}
\frac{\left\|\boldsymbol{t}-\boldsymbol{t}_{h}\right\|_{0, r}^{2}}{\delta+\|\boldsymbol{t}\|_{0, r}^{2-r}+\left\|\boldsymbol{t}_{h}\right\|_{0, r}^{2-r}} & +\int_{\Omega}\left|\boldsymbol{A}(\boldsymbol{t})-\mathcal{A}\left(\boldsymbol{t}_{h}\right)\right|\left|\boldsymbol{t}-\boldsymbol{t}_{h}\right| d x \\
& \leq \frac{\varepsilon^{r^{\prime}}}{r^{\prime}} \int_{\Omega}\left|\mathcal{A}(\boldsymbol{t})-\boldsymbol{\mathcal { A }}\left(\boldsymbol{t}_{h}\right)\right|\left|\boldsymbol{t}-\boldsymbol{t}_{h}\right| d x+\frac{C}{r \varepsilon^{r}}\left(\left\|\boldsymbol{t}-\boldsymbol{t}_{h}^{*}\right\|_{0, r}^{r}+\left\|\boldsymbol{\omega}-\boldsymbol{\omega}_{h}^{*}\right\|_{0, r}^{r}\right) \\
& +C\left(\left\|\boldsymbol{t}-\boldsymbol{t}_{h}^{*}\right\|_{0, r}+\left\|\boldsymbol{\omega}-\boldsymbol{\omega}_{h}^{*}\right\|_{0, r}\right)\left\|\boldsymbol{\sigma}-\boldsymbol{\sigma}_{h}^{*}\right\|_{0, r^{\prime}} \\
& +C\left\|\boldsymbol{t}-\boldsymbol{t}_{h}\right\|_{0, r}\left\|\boldsymbol{\sigma}-\boldsymbol{\sigma}_{h}^{*}\right\|_{0, r^{\prime}} .
\end{aligned}
$$

Choosing $0<\varepsilon<1$ (for example, $\varepsilon=1 / 2$ ), it follows from the previous inequality the following one:

$$
\begin{aligned}
\frac{\left\|\boldsymbol{t}-\boldsymbol{t}_{h}\right\|_{0, r}^{2}}{\delta+\|\boldsymbol{t}\|_{0, r}^{2-r}+\left\|\boldsymbol{t}_{h}\right\|_{0, r}^{2-r}} & +\int_{\Omega}\left|\mathcal{A}(\boldsymbol{t})-\mathcal{A}\left(\boldsymbol{t}_{h}\right)\right|\left|\boldsymbol{t}-\boldsymbol{t}_{h}\right| d x \\
& \leq C\left(\left\|\boldsymbol{t}-\boldsymbol{t}_{h}^{*}\right\|_{0, r}^{r}+\left\|\boldsymbol{\omega}-\boldsymbol{\omega}_{h}^{*}\right\|_{0, r}^{r}+\left\|\boldsymbol{t}-\boldsymbol{t}_{h}\right\|_{0, r}\left\|\boldsymbol{\sigma}-\boldsymbol{\sigma}_{h}^{*}\right\|_{0, r^{\prime}}\right. \\
& \left.+\left(\left\|\boldsymbol{t}-\boldsymbol{t}_{h}^{*}\right\|_{0, r}+\left\|\boldsymbol{\omega}-\boldsymbol{\omega}_{h}^{*}\right\|_{0, r}\right)\left\|\boldsymbol{\sigma}-\boldsymbol{\sigma}_{h}^{*}\right\|_{0, r^{\prime}}\right) .
\end{aligned}
$$

Then, using the stability conditions on $\boldsymbol{t}$ and $\boldsymbol{t}_{h}$ (see Theorem 3.1 and Theorem 4.1), we get, for all $\bar{\varepsilon}>0$,

$$
\begin{aligned}
\left\|\boldsymbol{t}-\boldsymbol{t}_{h}\right\|_{0, r}^{2} \leq & C\left(\left\|\boldsymbol{t}-\boldsymbol{t}_{h}^{*}\right\|_{0, r}^{r}+\left\|\boldsymbol{\omega}-\boldsymbol{\omega}_{h}^{*}\right\|_{0, r}^{r}+\left\|\boldsymbol{t}-\boldsymbol{t}_{h}\right\|_{0, r}\left\|\boldsymbol{\sigma}-\boldsymbol{\sigma}_{h}^{*}\right\|_{0, r^{\prime}}\right. \\
& \left.+\left(\left\|\boldsymbol{t}-\boldsymbol{t}_{h}^{*}\right\|_{0, r}+\left\|\boldsymbol{\omega}-\boldsymbol{\omega}_{h}^{*}\right\|_{0, r}\right)\left\|\boldsymbol{\sigma}-\boldsymbol{\sigma}_{h}^{*}\right\|_{0, r^{\prime}}\right) \\
\leq & C\left(\left\|\boldsymbol{t}-\boldsymbol{t}_{h}^{*}\right\|_{0, r}^{r}+\left\|\boldsymbol{\omega}-\boldsymbol{\omega}_{h}^{*}\right\|_{0, r}^{r}+\left(\left\|\boldsymbol{t}-\boldsymbol{t}_{h}^{*}\right\|_{0, r}+\left\|\boldsymbol{\omega}-\boldsymbol{\omega}_{h}^{*}\right\|_{0, r}\right)\left\|\boldsymbol{\sigma}-\boldsymbol{\sigma}_{h}^{*}\right\|_{0, r^{\prime}}\right) \\
& +\bar{\varepsilon}\left\|\boldsymbol{t}-\boldsymbol{t}_{h}\right\|_{0, r}^{2}+\frac{C}{\bar{\varepsilon}}\left\|\boldsymbol{\sigma}-\boldsymbol{\sigma}_{h}^{*}\right\|_{0, r^{\prime}}^{2},
\end{aligned}
$$

which implies (taking, for example, $\bar{\varepsilon}=1 / 2$ )

$$
\begin{aligned}
\left\|\boldsymbol{t}-\boldsymbol{t}_{h}\right\|_{0, r}^{2} \leq \quad & C\left(\left\|\boldsymbol{t}-\boldsymbol{t}_{h}^{*}\right\|_{0, r}^{r}+\left\|\boldsymbol{\omega}-\boldsymbol{\omega}_{h}^{*}\right\|_{0, r}^{r}+\left\|\boldsymbol{\sigma}-\boldsymbol{\sigma}_{h}^{*}\right\|_{0, r^{\prime}}^{2}\right. \\
& \left.+\left(\left\|\boldsymbol{t}-\boldsymbol{t}_{h}^{*}\right\|_{0, r}+\left\|\boldsymbol{\omega}-\boldsymbol{\omega}_{h}^{*}\right\|_{0, r}\right)\left\|\boldsymbol{\sigma}-\boldsymbol{\sigma}_{h}^{*}\right\|_{0, r^{\prime}}\right) .
\end{aligned}
$$

Thus, using the interpolation results and (25), we obtain

$$
\left\|\boldsymbol{t}-\boldsymbol{t}_{h}\right\|_{0, r} \leq C h^{m(r / 2)} .
$$

From (37) and this last estimate, we get

$$
\int_{\Omega}\left|\mathcal{A}(\boldsymbol{t})-\mathcal{A}\left(\boldsymbol{t}_{h}\right)\right|\left|\boldsymbol{t}-\boldsymbol{t}_{h}\right| d x \leq C h^{m r}
$$

and then, by (35) and Lemma 3.1, we have

$$
\begin{aligned}
\left\|\boldsymbol{\sigma}_{h}^{*}-\boldsymbol{\sigma}_{h}\right\|_{0, r^{\prime}} & \leq C\left(\left\|\mathcal{A}(\boldsymbol{t})-\mathcal{A}\left(\boldsymbol{t}_{h}\right)\right\|_{0, r^{\prime}}+\left\|\boldsymbol{\sigma}-\boldsymbol{\sigma}_{h}^{*}\right\|_{0, r^{\prime}}\right) \\
& \leq C\left(\left[\int_{\Omega}\left|\mathcal{A}(\boldsymbol{t})-\mathcal{A}\left(\boldsymbol{t}_{h}\right)\right|\left|\boldsymbol{t}-\boldsymbol{t}_{h}\right| d x\right]^{1 / r^{\prime}}+\left\|\boldsymbol{\sigma}-\boldsymbol{\sigma}_{h}^{*}\right\|_{0, r^{\prime}}\right) \leq C h^{m\left(r / r^{\prime}\right)}
\end{aligned}
$$

thus

$$
\left\|\boldsymbol{\sigma}-\boldsymbol{\sigma}_{h}\right\|_{0, r^{\prime}} \leq C h^{m\left(r / r^{\prime}\right)}=C h^{m(r-1)} .
$$

On the other hand, due to (30) and (23), we have

$$
\left\|p_{h}^{*}-p_{h}\right\|_{0, r^{\prime}} \leq C\left\|\boldsymbol{\sigma}_{h}^{*}-\boldsymbol{\sigma}_{h}\right\|_{0, r^{\prime}}
$$


and then we get the following estimation for the pressure

$$
\left\|p-p_{h}\right\|_{0, r^{\prime}} \leq C h^{m(r-1)}
$$

It remains to prove the error estimates (28) and (29). To this end, using (31) and the discrete Inf-Sup condition (22), we have

$$
\beta_{2}^{*}\left\|\left(\boldsymbol{u}_{h}^{*}-\boldsymbol{u}_{h}, \boldsymbol{\omega}_{h}^{*}-\boldsymbol{\omega}_{h}\right)\right\|_{M} \leq\left\|\boldsymbol{t}-\boldsymbol{t}_{h}\right\|_{0, r}+\left\|\boldsymbol{\omega}-\boldsymbol{\omega}_{h}^{*}\right\|_{0, r} \leq C h^{m(r / 2)}
$$

and then

$$
\left\|\boldsymbol{u}_{h}^{*}-\boldsymbol{u}_{h}\right\|_{0, r}+\left\|\boldsymbol{\omega}-\boldsymbol{\omega}_{h}\right\|_{0, r} \leq C h^{m(r / 2)}
$$

Finally, (29) is a consequence of this last estimate and the interpolation results.

Theorem 4.3. Let $r \geq 2$ and $m=1,2$. Suppose $\boldsymbol{t} \in\left[W^{m, r}(\Omega)\right]^{2 \times 2}, \underset{\sim}{\boldsymbol{\sigma}}=(\boldsymbol{\sigma}, p) \in\left[W^{m, r^{\prime}}(\Omega)\right]^{2 \times 2} \times$ $W^{m, r^{\prime}}(\Omega)$, and $\underset{\sim}{\boldsymbol{u}}=(\boldsymbol{u}, \boldsymbol{\omega}) \in\left[W^{1, r}(\Omega)\right]^{2} \times\left[W^{m, r}(\Omega)\right]^{2 \times 2}$. Then, there exists a positive constant $C$ independent of $h$ such that

$$
\begin{aligned}
&\left\|\boldsymbol{t}-\boldsymbol{t}_{h}\right\|_{0, r, \Omega} \leq C h^{m\left(r^{\prime}-1\right)}, \\
&\left\|\boldsymbol{\sigma}-\boldsymbol{\sigma}_{h}\right\|_{0, r^{\prime}, \Omega}+\left\|p-p_{h}\right\|_{0, r^{\prime}, \Omega} \leq C h^{m\left(r^{\prime} / 2\right)}, \\
&\left\|\boldsymbol{u}_{h}^{*}-\boldsymbol{u}_{h}\right\|_{0, r, \Omega}+\left\|\boldsymbol{\omega}-\boldsymbol{\omega}_{h}\right\|_{0, r, \Omega} \leq C h^{m\left(r^{\prime}-1\right)}, \\
&\left\|\boldsymbol{u}-\boldsymbol{u}_{h}\right\|_{0, r, \Omega} \leq C h^{r^{\prime}-1} \quad \text { if } m=1, \quad \text { and }\left\|\boldsymbol{u}-\boldsymbol{u}_{h}\right\|_{0, r, \Omega} \leq C h^{\min \left\{1,2\left(r^{\prime}-1\right)\right\}} \quad \text { if } m=2 .
\end{aligned}
$$

Proof. The proof of this last Theorem is similar to the one of Theorem 4.2.

\section{Conclusion}

We have developed in this paper a mixed approach for the approximation of all the important variables (stress, velocity and pressure) appearing in quasi-Newtonian fluid flow problems. Our approach allows to treat, independently, both the famous laws of Power and Carreau without using the inversion of constitutive laws. The continuous and discrete problems are well posed. The error estimates are optimal and are the same as the ones that we have obtained in the particular case of Power law (see Farhloul and Zine [9]).

\section{References}

[1] J. Baranger, K. Najib, Analyse numérique des écoulements quasi-newtoniens dont la viscosité obéit à la loi puissance ou la loi de Carreau, Numer. Math. 58 (1990) 35-49.

[2] J.W. Barrett, W.B. Liu, Finite element error analysis of a quasi-Newtonian flow obeying the Carreau or power law, Numer. Math. 64 (1993) 433-453.

[3] J.W. Barrett, W.B. Liu, Quasi-norm error bounds for the finite element approximation of non-Newtonian flow, Numer. Math. 68 (1994) 437-456.

[4] D. Boffi, F. Brezzi, M. Fortin. Mixed Finite Element Methods and Applications, Springer-Verlag, Berlin, Heidelberg, 2013.

[5] P.G. Ciarlet, The Finite Element Methods for Elliptic Problems, North Holland, 1978. 
[6] V.J. Ervin, J.S. Howell, I. Stanculescu. A dual-mixed approximation method for a three-field model of a nonlinear generalized Stokes problem, Clemson University Department of Mathematical Sciences Technical Report TR2007_08_EHS, 2007. $<$ http://www.math.clemson.edu/reports/TR2007_08_EHS.pdf $>$.

[7] V.J. Ervin, J.S. Howell, I. Stanculescu. A dual-mixed approximation method for a three-field model of a nonlinear generalized Stokes problem, Comput Methods Appl Mech Engrg 197 (2008) 2886-2900.

[8] M. Farhloul, A.M. Zine, A mixed finite element method for a Ladyzhenskaya model, Comput. Methods Appl. Mech. Engrg. 191 (2002) 4497-4510.

[9] M. Farhloul, A.M. Zine. A mixed finite element method for a quasi-Newtonian fluid flow, Numer Methods Partial Differential Eq 20 (2004) 803-819.

[10] M. Farhloul, A.M. Zine. A posteriori error estimation for a dual mixed finite element approximation of non-Newtonian fluid flow problems, International Journal of Numerical Analysis and Modeling 5 (2008) 320-330.

[11] G.N. Gatica, N. Heuer, S. Meddahi. On the numerical analysis of nonlinear twofold saddle point problems, IMA J. Numer. Analysis, 23 (2003) 301-330.

[12] G.N. Gatica, M. Gonzalez, S. Meddahi. A low-order mixed finite element method for a class of quasi-Newtonian Stokes flows. Part I: A priori error analysis, Comput Methods Appl Mech Engrg 193 (2004) 881-892.

[13] V. Girault, P.A. Raviart, Finite Element for Navier-Stokes Equations, Theory and Algorithms, Springer-Verlag, Berlin, 1986.

[14] O.A. Ladyzhenskaya, New equations for the description of the viscous incompressible fluids and solvability in the large of the boundary value problems for them, Boundary Value Problems of Mathematical Physics V. Providence, RI: American Mathematical Society, 1970.

[15] H. Manouzi, M. Farhloul, Mixed finite element analysis of a non-linear three fields Stokes model, IMA J. Numer. Anal, 21 (2001) 143-164.

[16] M. Renardy, R.C. Rogers, An introduction to Partial Differential Equations, Springer, New York, 1993.

[17] D. Sandri, Sur l'approximation numérique des écoulements quasi-Newtoniens dont la viscosité suit la loi puissance ou la loi de Carreau, M²AN 27 (1993) 131-155. 